Revue des patrimoines

$24 \mid 2014$

Architecture et urbanisme de villégiature : un état de la recherche

\title{
La villégiature retrouvée, nouvelles avancées et perspectives de la recherche (2004-2014)
}

\section{Claude Mignot}

\section{(2) OpenEdition \\ Journals}

Édition électronique

URL : http://journals.openedition.org/insitu/11197

DOI : $10.4000 /$ insitu. 11197

ISSN : 1630-7305

Éditeur

Ministère de la Culture

Référence électronique

Claude Mignot, "La villégiature retrouvée, nouvelles avancées et perspectives de la recherche

(2004-2014) », In Situ [En ligne], 24 | 2014, mis en ligne le 21 juillet 2014, consulté le 08 juillet 2020.

URL : http://journals.openedition.org/insitu/11197; DOI : https://doi.org/10.4000/insitu. 11197

Ce document a été généré automatiquement le 8 juillet 2020.

\section{cc) (†)}

In Situ Revues des patrimoines est mis à disposition selon les termes de la licence Creative Commons Attribution - Pas d'Utilisation Commerciale - Pas de Modification 4.0 International. 


\title{
La villégiature retrouvée, nouvelles avancées et perspectives de la recherche (2004-2014)
}

\author{
Claude Mignot
}

1 En 1953, dans Les vacances de M. Hulot, Jacques Tati célébrait avec son humour inimitable un mode de villégiature balnéaire familiale, que le double essor du transport aérien et des voyages lointains d'un côté, du tourisme de masse et des complexes urbains du littoral de l'autre, allait bientôt rendre désuet, comme sombraient dans la nostalgie les villages façon Jour de fête et la banlieue bon enfant de Mon oncle. Dans la France des Trente glorieuses, banlieues et villages traditionnels allaient connaître une profonde mutation dans un silence assourdissant; par contre, l'inflexion du tourisme (à vrai dire plus symbolique que réelle, car dans les années soixante les vacances ont continué presque comme avant dans ces stations familiales) favorisa l'émergence d'une historiographie balnéaire sophistiquée, la nostalgie ayant un effet heuristique ${ }^{1}$.

2 "L'histoire des stations balnéaires françaises a débuté il y a un peu plus de deux siècles ", écrit Bernard Toulier au tout début de la grande monographie qu'il a dirigée, Villégiature des bords de mer', mais l'histoire des stations balnéaires, dans un autre sens, c'est-à-dire l'historiographie de ces stations, a, elle, tout juste trente ans, ou quarante, si l'on veut faire bonne mesure et tenir compte de sa préhistoire.

3 Le bord de mer, qui était encore au XviII ${ }^{\text {e }}$ siècle le «territoire du vide » cher à Alain Corbin, inventeur d'une expression qui a fait florès, était devenu au cours du XIX $\mathrm{x}^{\mathrm{e}} \mathrm{le}$ cadre de toutes sortes d'expérimentations urbaines et architecturales, mais aussi sociales et esthétiques. C'était « le territoire du plein air marin », à la fois sanitaire et mondain. Après la Belle Époque, dans les années 1930, le tourisme balnéaire connut un nouveau pic: ce fut alors le temps des mondanités sportives, que connurent aussi montagne et villes d'eaux.

4 Dans les années 1960 , ce bord de mer saturé ne pouvait plus que « se défaire ", à l'image de la société qui l'avait inventé et déployé : il devenait un objet historiquement invisible. Les historiens de l'architecture ne cherchaient encore dans l'architecture du 
XIX ${ }^{e}$ siècle que les racines de la modernité, sans voir que la modernité était aussi dans cette conurbation linéaire des loisirs balnéaires. On célébrait «Paris, capitale du XIx ${ }^{e}$ siècle ", sans voir que tous les "Paris-Plage ${ }^{3}$ en étaient le complément nécessaire : le second volet du diptyque de la vie moderne.

L'histoire de la reconquête historiographique, qui nous a occupés une trentaine d'années, est maintenant bien établie avec ses jalons décennaux. Dix ans après le numéro fondateur de la revue Monuments historiques de 1974, dans lequel Bruno Foucart mettait en branle les recherches sur l'architecture du XIXe siècle, 1984 fut marqué par la publication du livre de Dominique Rouillard, Le Site balnéaire, qui jetait les bases de la problématique à partir du cas de la côte normande. En 1993, le premier bilan historiographique restait encore placé sous le signe de la marge (Bernard Toulier, "L'architecture des bains de mer, un patrimoine marginalisé ", Revue de l'art, n¹01, 1993), tandis que dix ans plus tard, en 2004, nous pouvions célébrer, déjà, une villégiature retrouvée $e^{4}$.

$\mathrm{Au}$ cours de cette dernière décennie, l'exploration monographique du rivage s'est encore poursuivie et précisée, mais les lignes de la recherche ne nous semblent pas avoir fondamentalement changé. Aussi avant d'aller plus avant, il n'est sans doute pas inutile de revenir rapidement sur l'antériorité.

\section{Une réhabilitation éditoriale}

Figure 1

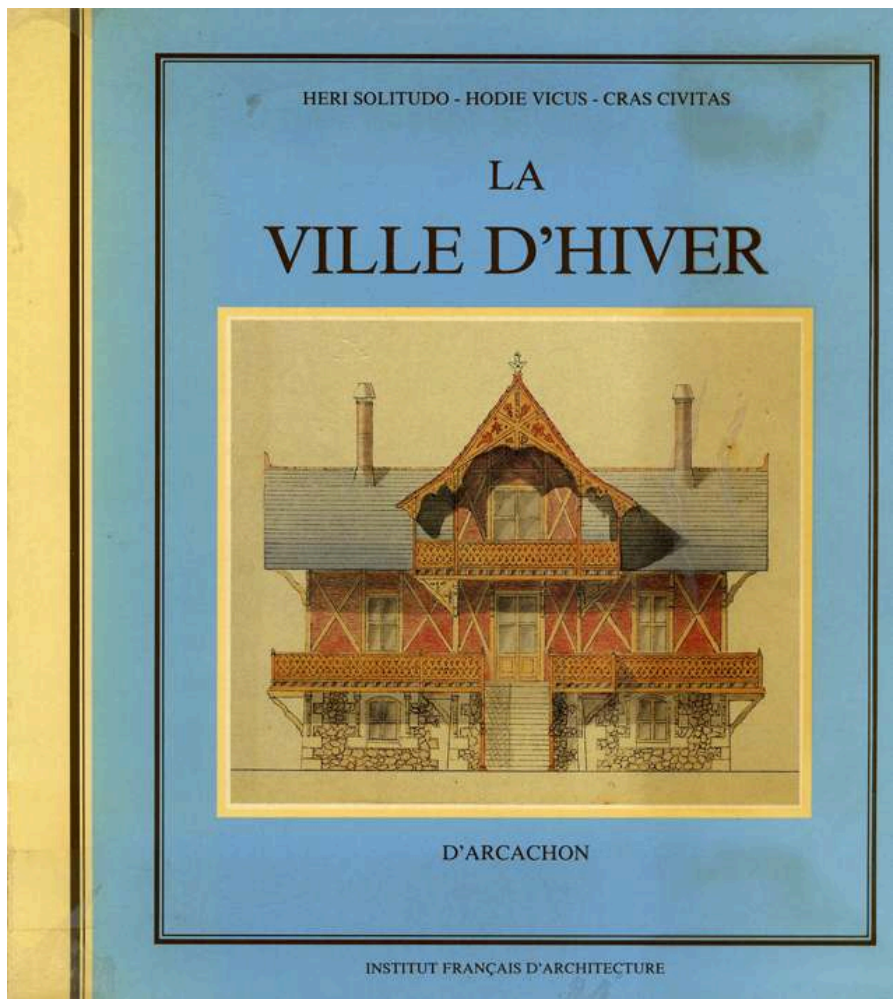

La ville d'hiver d'Arcachon. Paris : IFA, 1983.

Dès les années 1980, l'Institut français d'architecture (Ifa), sous l'impulsion de Maurice Culot, qui y dirigeait le département « Archives, histoire et documentation », s'était 
engagé dans une politique de publication de monographies de stations thermales et balnéaires, l'intérêt pour les secondes dérivant de la redécouverte des premières : après Vittel (1982), parurent ainsi aux éditions Mardaga La Ville d'hiver d'Arcachon (1983, nombreuses rééditions) (fig. 1), Trouville (1989), Architectures de Biarritz et de la Côte basque, de la Belle Époque aux années trente (1990). Parallèlement à l'exemple de l'Ifa, l'Inventaire général s'ouvrit à son tour à une exploration méthodique du rivage. Après les initiatives de Françoise Hamon qui organisa un premier stage sur l'architecture balnéaire pour les chercheurs de l'Inventaire général en 1988 et de Claude Laroche qui, sous la direction de Jean-Claude Lasserre, travaillait déjà sur Hossegor, le « Laboratoire de recherches sur le patrimoine français » (UMR 22) afficha en 1989, à mon initiative, l'architecture de villégiature comme thème prioritaire : ma participation à la monographie sur Trouville à l'invitation de Maurice Culot eut un rôle essentiel dans ce choix. Avec un certain retard, mais ensuite avec efficacité, certains universitaires, notamment Jean-Yves Andrieux à Rennes, suscitèrent des travaux de grande qualité. Les architectes et chercheurs de l'Ifa, les conservateurs et chargés d'études de l'Inventaire, mais aussi nombre de chercheurs indépendants, séduits de longue date par ce patrimoine, souvent spectaculaire, mais menacé par la spéculation et l'indifférence de certains services patrimoniaux, prirent l'habitude de collaborer. Dans les années qui suivirent s'établirent des croisements fructueux entre l'Ifa et l'Inventaire, notamment autour des éditions Norma, mais le ministère de la Culture s'engagea parallèlement, lui aussi, dans une grande politique éditoriale. À l'initiative de Bernard Toulier et avec le soutien de la direction du Patrimoine du ministère de la Culture puis également des régions d'accueil, des rencontres périodiques furent aussi organisées, permettant aux chercheurs de tous horizons de croiser recherches, expériences et résultats : Biarritz (23-25 septembre 1993), Cannes (20-22 avril 1994), Dinard (1995), Deauville (23-25 octobre 1997), Hyères (26-28 avril 1999), La Baule (14-17 octobre 2002), Vichy (18-21 octobre 2004), Mers-les-Bains (13-15 juin 2006), Soulac-sur-Mer (15 septembre 2007), Paris (7-9 octobre 2008), Biarritz (12-14 octobre 2010), Paris (à l'initiative de la Cité de l'architecture et du patrimoine, 8 juin 2011), Cerisy-la-Salle (10-17 juin 2013), Rouen (organisée par l'Institut national du patrimoine, 14-16 mai 2014).

7 En trente ans, depuis la publication de l'essai fondateur de Dominique Rouillard en 1984 jusqu'à aujourd'hui, en ce début d'année 2014, la « conurbation linéaire » des stations balnéaires françaises a été ainsi progressivement revisitée. Inventaires partiels de villas, recensements plus exhaustifs de stations, études approfondies de «côtes balnéaires ", trouvèrent les supports éditoriaux adaptés, d'une part auprès des éditions Norma, proches de l'Ifa, qui prirent le relais des éditions Mardaga, d'autre part dans trois collections liées à l'Inventaire général et à la direction du Patrimoine du ministère de la Culture : "Itinéraires du patrimoine», «Images du Patrimoine», "Cahiers de l'Inventaire", devenus "Cahiers du patrimoine». Les qualités visuelles de ces collections eurent un rôle pédagogique majeur auprès de tous les acteurs.

Du côté des éditions Norma et de l'Ifa, citons ainsi Claude Laroche, La côte landaise des années trente ; Hossegor 1920-1940, la station des sports élégants, Norma, 1991 ; Maurice Culot et Jacques Pavlovsky, La Côte basque des années trente; architectures d'André Pavlovsky, Norma, 1991; Maurice Culot (dir.) Biarritz. Villas et jardins. 1900-1930, Norma, 1992 ; Maurice Culot (dir.), La côte normande des années trente: Trouville-Deauville, société et architecture balnéaires 1910-1940, Norma, 1992 ; Maurice Culot et Bernard Toulier (dir.), Le 
Pays basque. Architectures des années 20 et 30, Norma, 1993 ; Richard Klein, Le Touquet Paris-Plage, Norma, 1994 ; Richard Klein (dir.), La Côte d'Opale : architecture des années 20 et 30, Norma, 1998.

Par un effet d'émulation positive, l'Inventaire publia une dizaine de petites anthologies de villas et de stations balnéaires dans la collection des "Itinéraires du patrimoine »: Jean-Louis Libourel, Villas de Houlgate $\left(\mathrm{n}^{\circ} 3\right), 1991$; Christine d'Aboville, Maguelonne Colonnier, Claire Étienne, Sainte-Adresse et le Nice havrais ( $\left.{ }^{\circ} 8\right), 1992$; Gilles Plum, Villas d'Arromanches-les-Bains: Asnelles, Tracy-sur-Mer, Ver-sur-Mer ( $\left.{ }^{\circ} 42\right), 1993$; Camille Milliet-Mondon, Cannes, architectures de villégiature $n^{\circ} 1\left(n^{\circ} 49\right), 1994$; Yannick Lecherbonnier, Hervé Pelvillain, Villas de Lions-sur-Mer et Hermanville-sur-Mer ( $\left.{ }^{\circ} 125\right)$, 1996 ; Geneviève Négrel, Hyères, la ville climatique ( $\left.{ }^{\circ} 126\right), 1996$; Marie-Noëlle Tournoux, Deauville, les styles normands (n²12), 1999 ; Bénédicte Dujardin et Claire Étienne, SaintValér-en-Caux, 2001; Carmen Popescu, Villas de Cabourg (n²33), 2003. Les «Images du patrimoine " offraient, elles, une lecture plus complète d'un territoire polyvalent: François Fray, Geneviève Négrel, Hyères ( $\left.{ }^{\circ} 198\right), 2000$; Laurent del Rosso, Cap d'Ail $\left(n^{\circ} 220\right), 2003$. Enfin, les études approfondies trouvèrent leur place dans les «Cahiers de l'Inventaire " rebaptisés «Cahiers du patrimoine»: Claude Laroche, Hossegor: 1923-1939: architecture et identité régionale (n³2), 1993 (fig. 2) ; Pierre-Yves Corbel, « Malo-les-Bains... » dans Dunkerque : dunes, briques et béton, Lille (n41), 1995.

Figure 2

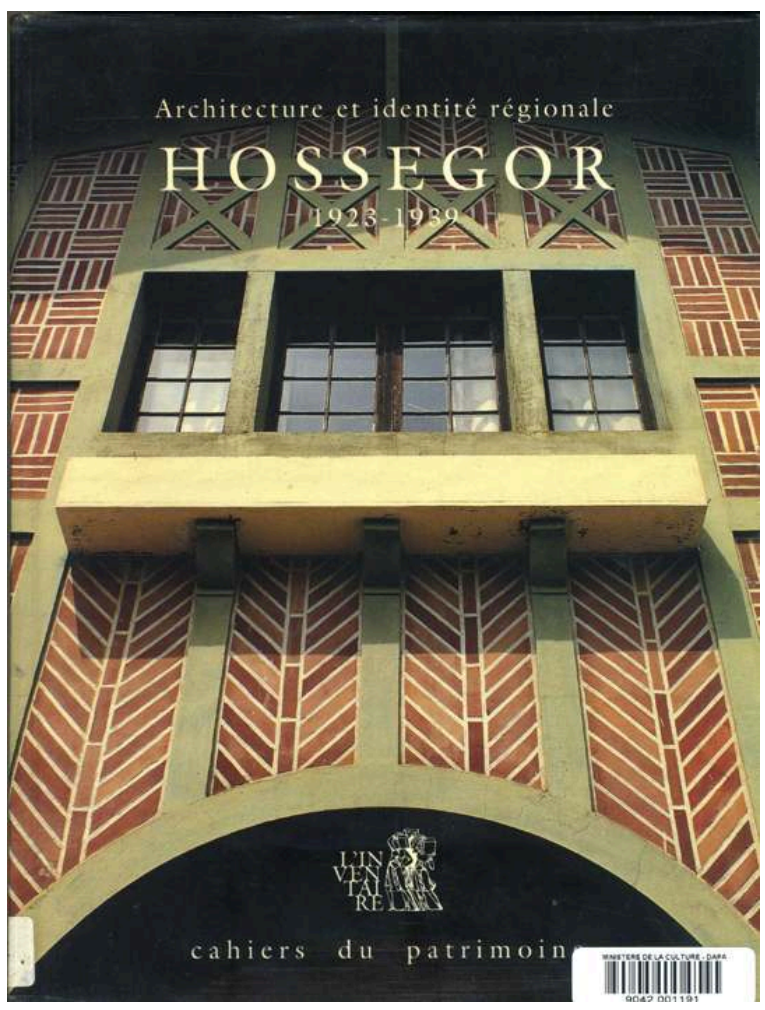

LAROCHE, Claude. Hossegor, 1923-1939. Architecture et identité régionale. Inventaire général des monuments et des richesses artistiques de la France, région Aquitaine. Bordeaux : A.P.I.A. ; Le Festin, 1993 (Coll. Cahiers du patrimoine, $n^{\circ}$ 32).

10 Quelques études extérieures à l'Ifa comme à l'Inventaire général furent publiées ailleurs. Elles ne bénéficièrent pas de l'effet de collection, qui donna corps au processus de redécouverte en cours, mais elles furent elles aussi portées par la vague ${ }^{5}$. Des 
ouvrages issus de thèses de doctorat furent accueillis par des presses universitaires, comme Gaëlle Delignon, Saint-Malo-Paramé. Urbanisme et architecture balnéaires. 1840-1940, PUR, 1999, ou des éditeurs privés, comme Nathalie Bertrand, Tamaris, entre Orient et Occident, Actes Sud, 2003. D'autres entrèrent dans la série des "Cahiers du patrimoine », comme Isabelle Barbedor (et alii), La Côte d'Émeraude (n60), 2001. On ne peut que regretter que cette politique n'ait pas été poursuivie pour la thèse d'Élisabeth Justome, Urbanisme et architecture balnéaire sur la Côte de Granit rose (Université de Rennes 2, 2005). Ce serait à l'honneur des collectivités locales de s'engager dans cette voie si l'État se retire des missions éditoriales dans lesquelles il s'était illustré pendant deux décennies.

11 On peut même regretter un certain reflux : alors que l'effet de série et de collection a joué un rôle décisif pour donner une image forte de cette redécouverte, la régionalisation a conduit à un éclatement des publications, en dépit des efforts faits pour maintenir une certaine cohérence éditoriale au-delà des diversités de financement.

\section{Vers un atlas de la conurbation balnéaire?}

Vers 2003, la masse des études partielles semblait permettre d'envisager le phénomène balnéaire sous une échelle globale, nationale, transcendant les études locales. En effet, si toutes les stations ont une dimension régionale attirant les baigneurs des villes les plus proches (Le Touquet/Lille ; Trouville-Deauville/Caen et Rouen; Dinard/Rennes ; La Baule et Pornic/Nantes; Arcachon/Bordeaux), la plupart des stations attirent des baigneurs de toute la France: la côte forme une conurbation linéaire en correspondance avec l'ensemble des villes du territoire, aussi bien Paris, que SaintRémy-sur-Deule, d'où part la famille Fenouillard pour prendre les bains de mer à SaintMalo ${ }^{6}$. Cependant la synthèse sur le phénomène balnéaire se fit attendre, et le projet d'«Atlas de la villégiature balnéaire», plusieurs fois envisagé, fut repoussé dans l'attente de nouvelles études.

13 Aussi, au cours de la dernière décennie, les recherches se sont principalement poursuivies sur la même ligne fragmentaire, régionale, voire locale: de nouvelles zones, de nouvelles stations ont été étudiées, ou réétudiées. Les travaux sur la Côte d'Opale se sont enrichis d'un beau recensement de la côte picarde par Élisabeth Justome, dont les résultats sont maintenant publiés en ligne, tandis qu'une modeste station familiale comme Le Crotoy, sur la baie de Somme, a fait l'objet d'une première étude fouillée, laquelle a mis en évidence un type original de station où les villas balnéaires se sont insérées dans le tissu ancien de maisons de pêcheurs au lieu de se juxtaposer dans un quartier des bains, comme à Trouville par exemple (Annie Jacques, La Vie balnéaire en baie de Somme, Le Crotoy au temps des Guerlain, Jules Verne, Colette et Toulouse-Lautrec, Engelaere Éditions, 2011). Enfin, vient de paraître Berck-sur-mer, du soin à la villégiature, Nord-Pas-de-Calais dans la collection des « Images du patrimoine » (Lieux Dits, 2014).

14 Une première monographie sur Dieppe (Viviane Manase, Dieppe, Patrimoine et territoire, $\mathrm{n}^{\circ} 1,2010$ ) et une mise à jour des recherches sur «le Nice havrais » (Claire Étienne et Christine d'Aboville, Sainte-Adresse et le Nice havrais, Patrimoine et territoire, $\mathrm{n}^{\circ} 2$, 2010) constituent un embryon prometteur d'une étude complète de la Côte d'Albâtre. Il était important en effet de redécouvrir l'importance de cette côte avec son 
paysage de falaises et de valleuses, où se nichent les stations. Dieppe, comme Boulogne, fait partie de la première génération des grandes stations « mondaines", mais il fallait (et il faudra encore) étudier aussi les stations familiales, comme les Petites Dalles, Saint-Pierre-en-Port, Yport, de part et d'autre de Fécamp, qui attiraient et attirent toujours en grand nombre artistes, écrivains et musiciens.

Le rivage de Basse-Normandie, premier segment constitué de la conurbation balnéaire, méritait de faire l'objet d'une étude globale. Le catalogue de l'exposition de Caen (Alice Gandin [dir.], Destination Normandie. Deux siècles de tourisme, $\mathrm{XIX}^{e}-\mathrm{XX} \mathrm{X}^{e}$ siècles, 5 Continents Éditions, 2009) offrit des approches suggestives. De son côté, Gilles Plum proposa une anthologie commentée (À l'apogée de la villa, Côte Fleurie, 1870-1920, Cahiers du Temps, $2^{2007)^{7}}$. Mais ni l'un ni l'autre ne visait une synthèse complète. La Côte Fleurie continue donc d'être approchée station après station, ce qui ne permet pas encore de mesurer l'ampleur du phénomène culturel et architectural.

Se précise cependant la connaissance des principales stations, Trouville, Deauville, Cabourg, Houlgate, dont chacune mériterait comme Le Touquet de s'appeler « ParisPlage ». Citons notamment Didier Hébert, Deauville, une cité de villégiature de la Côte Fleurie, Images du patrimoine, 2004, et, du même auteur, Houlgate, la perle de la Côte Fleurie, Parcours du patrimoine, nouveau nom de la collection des «Itinéraires" (n³11), 2011.

17 Entre La Baule, objet d'une monographie déjà ancienne (Alain Charles, La Baule et ses villas, 2002), et Royan, plus récemment étudiée (Thierry Jeanmonod, Nicolas Nogue, Chantal Callais, Gilles Ragot, L'invention d'une ville; Royan années 50, Cahiers du patrimoine, $\left.n^{\circ} 65,2003\right)$, est venue s'ajouter une monographie sur la Côte de Jade (Agathe Aoustin, Urbanisme et architecture balnéaire de la Côte de Jade, 1820-1975 (thèse de doctorat, université de Paris IV, sous la direction de Jean-Yves Andrieux, 2013) et sur Les Sables-d'Olonne (Louise Robin, Michel Brossard, Alain-Pierre Daguin, Villas et édifices balnéaires des Sables-d'Olonne, un siècle d'architecture, 1845-1945, éditions de Beaupré, 2011). Plus au sud, sur la côte landaise autour d'Hossegor et sur la Côte basque autour de Biarritz, de nouvelles publications sont venues conforter ce qui était solidement acquis (Claude Laroche, Hossegor, Itinéraires du patrimoine, $n^{\circ} 294,2004$ ).

En dépit des études pionnières sur Cannes et Hyères, la Côte d'Azur résiste. On peut cependant relever quelques études exemplaires: Geneviève Négrel, Saint-Raphaël (Cahiers de la médiathèque, $\mathrm{n}^{\circ} 4$ ), 2009 ; Nathalie Rosticher Giordano (dir.), Monacopolis. Architecture, Urbanisme et Urbanisation à Monaco. Réalisations et projets. 1858-2012, Nouveau musée national de Monaco, 2013. Si aujourd'hui on reste encore loin d'une étude complète du linéaire littoral, et donc de la possibilité d'établir un atlas définitif de la "conurbation balnéaire", les principaux pôles de cette conurbation sont donc maintenant explorés et ont fait l'objet de publications majeures.

\section{Du balnéaire à la montagne et au suburbain : une ouverture des perspectives}

Sur ce fond de continuité, on voit cependant se dessiner quelques lignes de force.

$\mathrm{Au}$ cours de la dernière décennie, on a vu s'approfondir l'étude du phénomène balnéaire aux deux extrémités de son histoire. La préhistoire des stations a mis en évidence une multipolarité, un peu inattendue, du phénomène. Si l'importance 
pionnière des touristes anglais sur les côtes françaises a été confirmée à Boulogne-surMer et Dieppe, d'autres sites méconnus sont apparus, comme la côte vendéenne, la future Côte de Jade, avec une dimension thermale: («l'eau ferrugineuse », rendue célèbre par un sketch de Bourvil).

En phase avec le développement des recherches sur l'architecture $d u x^{e}$ siècle au sein de l'Inventaire général, on a vu aussi l'essor des recherches sur la villégiature contemporaine, pas seulement celle des années 1930, mais aussi celle des Trente glorieuses. Dans le numéro spécial de la Revue de l'art $\left(n^{\circ} 165,2009\right)$, coordonné par JeanYves Andrieux, sur la villégiature balnéaire contemporaine, Richard Klein revisitait Le Touquet au temps des Trente glorieuses. Dans ce numéro-ci, Jean-François Pinchon propose une approche d'ensemble du phénomène des stations nouvelles du LanguedocRoussillon, approche que Michel Marty développe pour la compréhension de l'aménagement de la côte aquitaine à partir des années 1970. Bertrand Charneau dépasse quant à lui la chronologie habituelle des études sur Arcachon. Enfin, toujours pour ces mêmes périodes récentes, Jacques Labro et Franck Delorme, l'acteur et l'historien, donnent, chacun avec ses mots, des clés de compréhension pour le développement de la villégiature en montagne et les formes qu'elle a prises.

Les travaux sur l'architecture de la villégiature balnéaire s'étaient initialement développés conjointement aux études sur l'architecture thermale; depuis près de vingt-cinq ans, le parallèle est aussi établi avec la villégiature suburbaine, de bord de fleuve et de forêt. Les tout premiers critiques des stations balnéaires avaient bien noté le lien entre les chalets suburbains d'île-de France et les villas de Trouville ${ }^{8}$. Dans Le Vésinet, modèle français d'urbanisme paysager (Cahiers de l'Inventaire, n¹7), 1989, Sophie Cueille en fit une démonstration qui fit date. L'heureuse expression lancée, dans la préface de cet ouvrage, par François Loyer, « la villégiature de bord de ville », est venue conforter la réflexion conduite sur la spécificité des villages d'île-de-France dès l'Ancien Régime par l'équipe de l'Inventaire d'île-de-France. Le lien s'est ensuite aussi établi entre les villégiatures balnéaire et cynégétique, comme le fit aussi Bernard Toulier dans Châteaux en Sologne en 1992...

Comme le montre ici de façon convaincante Roselyne Bussière, les études se sont développées en Île-de-France, sous l'impulsion et la direction de Dominique Hervier, après la monographie pionnière sur le Vésinet: Laurent Robert, Chatou, Croissy-surSeine, Villégiatures en bord de Seine (Images du patrimoine, n¹28), 1993 ; Roselyne Bussière, Saint-Germain-en-Laye, 1800-1940 (Images du patrimoine, $\mathrm{n}^{\circ} 159$ ), 1997 ; Laurence de Finance, Clamart, une ville à l'orée du bois (Images du Patrimoine, ${ }^{\circ} 164$ ), 1997 ; Sophie Cueille, Maisons-Laffitte, parc, paysage et villégiature, 1630-1930 (Cahiers du patrimoine, $\mathrm{n}^{\circ} 53$ ), 1999 ; Roselyne Bussière et alii, Au sud de Versailles. Buc, Jouy-en-Josas, Les Loges-en-Josas, Toussus-Le-Noble (Images du patrimoine, $\mathrm{n}^{\circ} 210$ ), 2001.

Les publications se poursuivirent après 2004: Isabelle Duhau, Autour d'Orgeval, de la boucle de Poissy au pays de Cruye (Images du patrimoine, n²00), 2002, rééd. 2008 ; Nogent et Le Perreux, l'Eldorado des bords de Marne (Images du patrimoine, n²37), 2005 ; Isabelle Duhau et Laurence de Finance, Bry et Champigny dans les méandres de la Marne (Images du patrimoine, $\mathrm{n}^{\circ} 247$ ), 2007 ; Roselyne Bussière, Un belvédère en Yvelines, Andrésy (Images du patrimoine, $\left.\mathrm{n}^{\circ} 252\right), 2008$; Sophie Cueille, Enghien-les-Bains, architecture et décor (Images du patrimoine, $\left.\mathrm{n}^{\circ} 255\right), 2010$.

Comme on l'a déjà suggéré, il faut également ajouter, dans cette quête des différents types de villégiature, les études sur la montagne et singulièrement celles de la région 
Rhône-Alpes, illustrées en 2012 par un ouvrage sur les stations de sports d'hiver: Maryannick Chalabi, Jean-François Lyon-Caen, Stations de sports d'hiver: urbanisme et architecture, hors collection, Lieux Dits. L'ouvrage et l'exposition qui l'accompagnait montrent toute l'ampleur d'un phénomène qui n'est plus seulement limité aux rivages maritimes. C'est d'ailleurs peut-être au beau livre de Françoise Véry et Pierre Saddy sur l'architecte Henri Jacques Le Même que l'on doit d'avoir ouvert la voie dès les années $1980^{\circ}$.

Ce sont aussi les méthodes qui ont évolué au cours de cette dernière décennie. On a vu se préciser les approches cartographiques et quantitatives. Le phénomène balnéaire étant un phénomène spatial de masse, il apparut bientôt que la carte était le moyen le plus efficace, le plus parlant et le plus synthétique, bref, le mieux à même de traduire les faits sur un linéaire côtier important. À cet égard, la publication sur la Côte d'Émeraude nous paraît avoir valeur de modèle. On mesure plus précisément aussi l'influence des guides touristiques dans la construction de l'espace balnéaire: Dominique Rouillard l'avait mise en évidence; nous l'avons démontrée à partir de l'exemple des villas de Trouville; l'approche a été généralisée ${ }^{10}$. Bernard Toulier et d'autres ont aussi souligné au temps de l'âge d'or du service des Postes la valeur documentaire exceptionnelle des cartes postales ${ }^{11}$.

Ces diverses avancées devaient permettre d'envisager une nouvelle étape. Depuis l'essai fondateur de Dominique Rouillard en 1984, personne n'avait proposé d'analyse d'ensemble, hors les bilans historiographiques de Bernard Toulier en 1993 et de moimême en 2004, déjà cités. Or, au cours des cinq dernières années, de nouvelles analyses globales sont parues, sous des formats très différents. Mentionnons une première tentative synthétique sous la direction d'Alain Lottin et de Jean-Pierre Poussou, Les Villes balnéaires d'Europe occidentale $d u$ XVIII à nos jours, PUSP, 2008, actes d'un colloque tenu à Calais, synthèse pionnière, même si elle présente nécessairement les limites d'un volume d'actes, juxtaposant par nature des essais très divers.

Citons aussi deux articles, brefs, mais de visées panoramiques. Dans le numéro 165 de la Revue de l'art, déjà cité, le titre de l'éditorial de Jean-Yves Andrieux, « Le Site balnéaire » renvoie explicitement à l'ouvrage inaugural de Dominique Rouillard, mais le sous-titre marque le déplacement des lignes de recherches: "De l'invention d'un type architectural, urbain et paysager au souci patrimonial d'un mode de sociabilité et de loisirs ». De mon côté, dans les Mélanges en l'honneur de Jean-Pierre Poussou, le titre de ma contribution, «La Station balnéaire, une invention du XIX siècle », donne l'ambition de cette dizaine de pages, où j'ai cherché à ramasser les points les plus décisifs du phénomène, et d'abord sa contribution à la définition de la ville et de la vie modernes ${ }^{12}$.

Mais l'exposé majeur est naturellement le gros et beau volume collectif, dirigé par Bernard Toulier, Villégiature des bords de mer, architecture et urbanisme, XVIII -XXe siècle, soutenu par diverses collaborations des services régionaux de l'Inventaire et publié en 2010 aux Éditions du patrimoine (fig. 3). Il s'agit du premier volume de la collection "Patrimoines en perspective », portée par la Direction générale des patrimoines, dont le nom indique bien éditorialement la reconnaissance, construite au cours de trois décennies, du patrimoine balnéaire. L'ouvrage, qui devrait servir de point de départ à une importante exposition de la Cité de l'architecture et du patrimoine, prévue pour 2016, est à ce jour la tentative la plus ambitieuse de ramasser en un volume l'essentiel des acquis de trente ans de recherches sur l'ensemble du phénomène. Nous invitons donc le lecteur à s'y reporter d'urgence : les grandes lignes sont maintenant solidement 
fixées, illustrées par les exemples les plus éclairants, servies par une iconographie flamboyante.

Figure 3

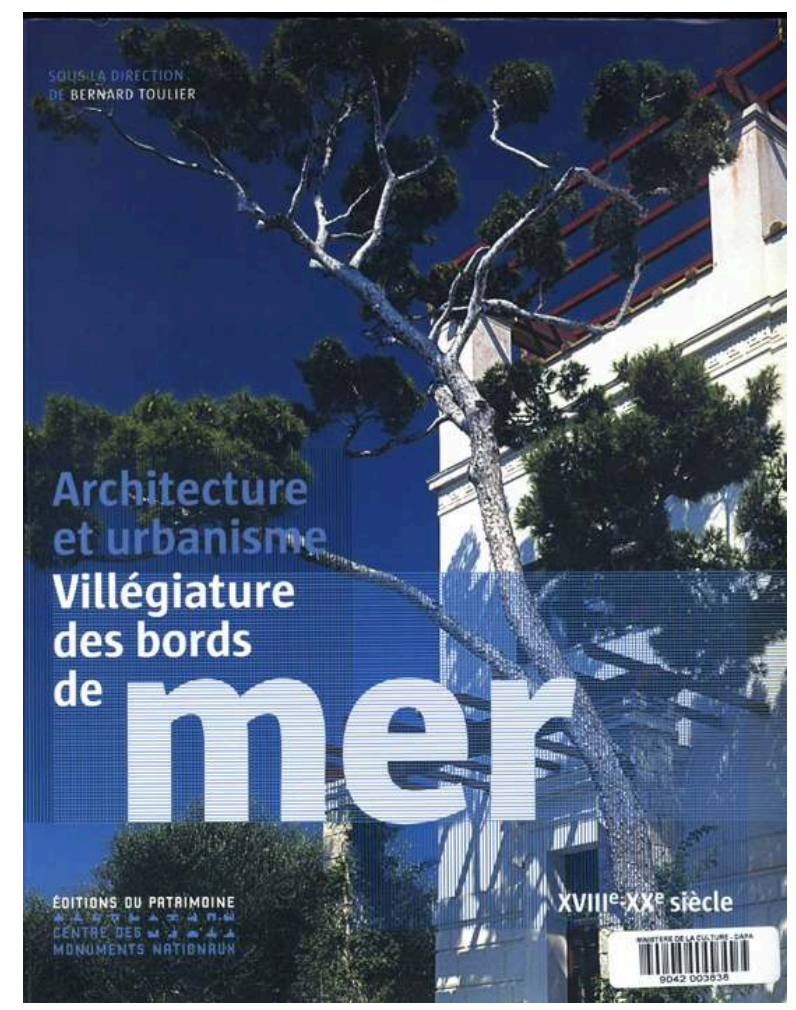

TOULIER, Bernard (dir.). Villégiature des bords de mer, architecture et urbanisme, $x \mathrm{VIII} \mathrm{e}^{\mathrm{e}} \mathrm{xx} \mathrm{e}^{\mathrm{e}}$ siècle. Paris : Éditions du patrimoine, 2010 (coll. Patrimoines en perspective).

\section{NOTES}

1. - Ce texte offre une version profondément remaniée - et mise à jour au début de l'année 2014 de notre intervention «La Villégiature retrouvée, avancées et perspectives de la recherche (2003-2011) », pour la journée d'étude « De la villégiature aux lieux de loisirs », Cité de l'architecture et du patrimoine, 11 juin 2011. Il actualise et prolonge le texte d'introduction du précédent numéro d'In Situ consacré à la villégiature (MIGNOT, Claude. «La Villégiature retrouvée : les réseaux de la recherche ». In Situ [En ligne], ${ }^{\circ} 4,2004$, [consulté le 08 juillet 2014]. URL : http:// insitu.revues.org/1289). Pour ses observations pertinentes, qui nous ont permis d'amender une première version de ce texte, nous remercions vivement Dominique Hervier, qui publie un article parallèle pour un dossier «Villégiature » dans la revue Histoire urbaine, 2014-IV, à paraître fin 2014.

2. - TOULIER, Bernard (dir.). Villégiature des bords de mer, architecture et urbanisme, XVIII ${ }^{e}-X X^{e}$ siècle. Paris : Éditions du patrimoine, 2010 (coll. Patrimoines en perspective). 
3. - La station du Touquet est qualifiée explicitement de Paris-Plage, mais les côtes normande, bretonne et basque offraient chacune implicitement autant de « Paris-Plage ».

4. - MIGNOT, Claude. « La Villégiature retrouvée : les réseaux de la recherche », op. cit.

5. - En 2001, le $36^{\mathrm{e}}$ Congrès de la Fédération des sociétés historiques et archéologiques de Normandie, tenu à Trouville, fut consacré au sujet: Bains de mer et thermalisme en Normandie (Annales de Normandie, vol. 7, 2002).

6. - CHRISTOPHE. La Famille Fenouillard. Paris : 1893 et 1895 (rééd. Armand Colin, 1984).

7. - Voir aussi PLUM, Gilles. Trouville, Deauville et Côte Fleurie; villas balnéaires du Second Empire, 1850-1870. Caen : Cahiers du Temps, 2007.

8. - Voir MIGNOT, Claude. «Les Villas, vrais monuments de Trouville». Dans CULOT, Maurice, JAKOVLJEVIC, Nada. Trouville. Bruxelles : Mardaga, 1989.

9. - VÉRY, Françoise, SADDY, Pierre. Henry Jacques Le Même, architecte à Megève. Bruxelles: Mardaga, 1988 ; CULOT, Maurice, LAMBRICHS, Anne. Megève 1925-1950. Architectures d'Henry Jacques Le Même. Paris : Norma, 1999 ; DELORME, Franck. Architecture de Henry Jacques Le Même. Répertoire des archives de l'architecte. Chambéry: Assemblée des Pays de Savoie, 2005.

10. - TOULIER, Bernard. L'influence des guides touristiques dans la représentation et la construction de l'espace balnéaire (1850-1950). Voir le site : http://www.culture.gouv.fr/culture/inventai/telechar/ toulier.pdf.

11. - TOULIER, Bernard. «L'Influence des guides touristiques dans la représentation et la construction de l'espace balnéaire (1850-1950)». Dans CHABAUD, Gilles, COHEN, Évelyne, COQUERY, Natacha, PENEZ, Jérôme (dir.). Les Guides imprimés $d u X I^{e}$ au XXe siècle. Villes, paysages, voyages. Paris : Belin, 2000, p. 239-258.

12. - Dans Les Passions d'un historien; mélanges en l'honneur de Jean-Pierre Poussou. Paris : PUPS, 2010, p. 1077-1087.

\section{AUTEUR}

\section{CLAUDE MIGNOT}

Professeur émérite à l'université de Paris-Sorbonne claude.mignot@paris-sorbonne.fr 